\title{
EARLY-STAGE MODELLING AND FORECAST OF COVID-19 OUTBREAK IN BURKINA FASO USING A BAYESIAN SIR APPROACH
}

\section{Serge Manituo Aymar Somda1, Eric Bernard Agodio Dabone ${ }^{2}$, Moussa Doulougou ${ }^{3}$, Cedric Stéphane Bationo ${ }^{4}$ and Kenneth Tiemoko Marie Galboni ${ }^{5}$}

${ }^{1}$ UFR/ST, Université Nazi Boni, Bobo-Dioulasso, Burkina Faso.

Centre MURAZ, Institut National de Santé Publique, Bobo-Dioulasso, Burkina Faso. E-mail:manituo@gmail.com

${ }^{2}$ UFR/ST, Université Nazi Boni, Bobo-Dioulasso, Burkina Faso.

Centre MURAZ, Institut National de Santé Publique, Bobo-Dioulasso, Burkina Faso.

${ }^{3}$ UFR/SATIC, Université Alioune Diop de Bambey, Sénégal.

Centre MURAZ, Institut National de Santé Publique, Bobo-Dioulasso, Burkina Faso.

${ }^{4}$ Aix Marseille Univ, INSERM, IRD, SESSTIM, UMR1252, ISSPAM, 13005 Marseille, France.

${ }^{5}$ UFR/ST, Université Nazi Boni, Bobo-Dioulasso, Burkina Faso.

Centre MURAZ, Institut National de Santé Publique, Bobo-Dioulasso, Burkina Faso.

Cite this article:

Somda S.M.A., Dabone E.B.A., Doulougou M., Bationo C.S., Galboni K.T.M. (2021), Early-Stage Modelling and Forecast of COVID-19 Outbreak in Burkina Faso using a Bayesian SIR Approach. African Journal of Mathematics and Statistics Studies 4(3), 145-156. DOI: 10.52589/AJMSSY8NXO02C.

\section{Manuscript History \\ Received: 6 Oct 2021 \\ Accepted: 23 Oct 2021 \\ Published: 27 Nov 2021}

ABSTRACT: In this article, we propose a Bayesian approach for estimating and predicting the magnitude of the coronavirus epidemic in Burkina Faso in its early stage. Our approach is inspired by the work of Wang et al. but adapted to the Burkinabe context. Two models are presented: a simple Bayesian SIR approach and another Bayesian SIR which takes into account the public health measures undertaken by the government of Burkina Faso. The approach was implemented at the early stage of the COVID-19 pandemic in Burkina Faso, covering the period from March 9 to April 30, 2020. The results of the analyses will allow a good prediction of COVID-19 infections and deaths in the early days of the epidemic, considering government policies.

KEYWORDS: COVID-19, SIR Model, Bayesian Model, Burkina Faso, Africa. 


\section{INTRODUCTION}

The SARS-COV-2 virus human infection appeared in December 2019 in Wuhan, capital of the province of Hubei (C. Wang et al., 2020). The later named COVID-19 quickly spread in Hubei and then in China to spread to the rest of the world. It quickly evolved from the stage of a simple epidemic to that of global pandemic (Sohrabi et al., 2020).

Burkina Faso is a landlocked country in West Africa that covers an area of around 274,200 square kilometers. This country is considered as one of the poorest in the world. The country's human development index was estimated at 0.452 in 2019, with a rank of 182 out of 189 countries and territories (UNDP, 2020). In 2019, the life expectancy at birth was 61.6 years and the adult's literacy rate, $41.2 \%$. The economic level is low with an annual gross domestic production estimated at 2,190 USD (2017 PPP) per capita and $43.7 \%$ of the population were living below the income poverty line ( $\$ 1.90$ a day).

Burkina Faso recorded its first COVID-19 confirmed case on March 9, 2020 from a travelling couple from France (Thiombiano, 2020). On April 30, less than two months later, the country recorded 649 confirmed cases and 44 deaths. Among the confirmed cases, 517 had recovered while 132 were still under observation.

Like other countries in the world, Burkinabe decision-makers took early measures to contain the pandemic in the territory. These include among others, the closure of schools on March 16, 2020 (B24, 2020), the introduction of curfews and the closure of international borders on March 21, 2020 (lefaso.net, 2020). Further movement restriction measures were subsequently taken (AIB, 2020; Kindo, 2020). Public health measures were taken in a context where no curative or preventive treatment was known. Moreover, knowledge on the evolution of the epidemic was very low in the countries that were the first to be affected and almost non-existent in the countries of sub-Saharan Africa.

Early mathematical contributions were made to provide a better understanding of the infection (Erik, Volz et al., 2020; Imai, Cori et al., 2020; Z. Liu et al., 2020; Rabajante, 2020). Specific working groups provided insightful mathematical models for decision making (Ferguson et al., 2020; GU et al., 2020; Imai, Dorigatti, et al., 2020).

In the context of Burkina Faso, mathematical methods have also been developed to model the effects of public policies decided by the Burkinabe government on the spread of the virus (Guiro et al., 2020; Zio et al., 2020). However, the country soon found itself limited in terms of information for decision-making. The proposed models were generally based on very strong assumptions. Basic information about the epidemic such as infectiousness, transmission serial interval and duration of infection were not really known. Estimates were made in the literature but these concerned countries far from Burkina Faso where the health, demographic, economic context, climate, etc. were very different. The few pieces of information produced by mathematicians therefore lacked the reliability necessary for effective decision-making.

L. Wang et al. (2020) very early proposed for China a Bayesian statistical approach to estimate and to predict the magnitude of the epidemic in early phases. This approach can provide faster and more efficient decision-making tools. The objective of this work was to adapt the method to the Burkinabe context and to propose a good forecast of COVID-19 infections and deaths in the first days of the outbreak, taking into account government policies. 


\section{DESCRIPTION OF THE MODEL}

\section{Primary model}

The proposed approach uses Susceptible-Infectious-Removed (SIR) type modelling. We consider two time series representing the proportions of infected and removed cases in the population. They are denoted respectively by $Y_{t}^{I}$ and $Y_{t}^{R}$ at time t, where the compartment $\mathrm{R}$ (removed) is a sum of the proportions of cured cases and deaths at time $t$. L. Wang et al. (2020) proposed that the two-dimensional time series of $\left(Y_{t}^{I}, Y_{t}^{R}\right)$ be a state space model with beta distributions at time $\mathrm{t}$ :

$$
Y_{t}^{I}\left|\theta_{t}, \tau \sim \operatorname{Beta}\left(\lambda^{I} \theta_{t}^{I}, \lambda^{I}\left(1-\theta_{t}^{I}\right)\right) ; Y_{t}^{R}\right| \theta_{t}, \tau \sim \operatorname{Beta}\left(\lambda^{R} \theta_{t}^{R}, \lambda^{R}\left(1-\theta_{t}^{R}\right)\right)
$$

The dynamics of COVID-19 infection are characterized by a latent Markov process: $\theta_{t}=$ $\left(\theta_{t}^{S}, \theta_{t}^{I}, \theta_{t}^{R}\right), \theta_{t} \in[0 ; 1]^{3}$ which is a three-dimensional process defined by:

$\theta_{t} \mid \theta_{t-1}, \tau \sim \operatorname{Dirichlet}\left(k . f\left(\theta_{t-1}, \beta, \gamma\right)\right)$

where

$\theta_{t}^{I}=E\left(\theta_{t}\right), \theta_{t}^{R}=E\left(Y_{t}^{R} \mid \theta_{t}\right)$ and $\theta_{t}^{S}+\theta_{t}^{I}+\theta_{t}^{R}=1$

$\theta_{t}^{S}$ represents the probability for an individual to be susceptible or at risk at time $t, \theta_{t}^{I}$ represents the probability for an individual to be infected at time $t$, and $\theta_{t}^{R}$ represents the probability for an individual to be removed from the infectious compartment by being cured or deceased. The parameters $\lambda^{I}>0$ and $\lambda^{R}>0$ are the parameters controlling the respective variances of the observed proportions.

The parameter $k$ scales the variance of the Dirichlet distribution and the function $f($.$) is a$ three-dimensional vector that determines the mean of the Dirichlet distribution. The $f($. function is the driver of infection dynamics which works according to the classical SIR model of infectious diseases. It is obtained using the fourth order Runge-Kutta approximation (RK4).

The parameters $\beta$ and $\gamma$ represent the rate of spread of the disease and the rate of remission respectively. They are determined by solving the below equation:

$$
\left\{\frac{d S(t)}{d t}=-\beta \times \frac{S(t) I(t)}{N} \frac{d I(t)}{d t}=\beta \times \frac{I(t) S(t)}{d t} \frac{d R(t)}{d t}=\quad \gamma I(t)-\gamma I(t)\right.
$$

where

$S(t), I(t)$ and $R(t)$ represent respectively the populations of susceptible, infected and cured at time $t$. $\frac{S(t) I(t)}{N}$ is the probability of an encounter between a healthy person and an infected person. Once determined, these parameters are then introduced into the system:

$$
\left\{\frac{d \theta_{t}^{S}}{d t}=-\beta \theta_{t}^{S} \theta_{t}^{I} \frac{d \theta_{t}^{I}}{d t}=\beta \theta_{t}^{S} \theta_{t}^{I} \quad \frac{d \theta_{t}^{R}}{d t}=\quad \gamma \theta_{t}^{I}-\gamma \theta_{t}^{I}\right.
$$


The other parameters of the model designated by $\tau=\left(\theta_{0}, k, \lambda\right)^{T}$ are estimated by MCMC simulation.

The following assumptions are made for the model:

- (H1): The size of the population is equal to $N$ and considered fixed: demographic phenomena (births, deaths, immigration, emigration) are neglected.

- (H2): The only possibility for an individual to leave the healthy group is by becoming infected. The number of new cases over a given period of time is proportional to the number of contacts over that period between susceptible individuals and infected individuals $(S(t) \times I(t))$. We note this coefficient of proportionality $\beta$.

- (H3): Infected people are all infectious; they can transmit the disease.

- (H4): We admit that all withdrawn individuals acquire immunity to the disease; they can no longer get infected again.

- (H5): We hypothesize that anyone who gets sick eventually withdraws (heal or die).

\section{Consideration of public health actions to limit the epidemic}

A second approach is described to deal with the government's public health measures to limit the epidemic. We consider for any moment $t, q^{S}(t) \in[0 ; 1]$ the risk that a person is isolated at home (contact case), and $q^{I}(t) \in[0 ; 1]$ the risk that an infected person is quarantined in the hospital. Thus, the risk of disease transmission when a person at risk encounters an infected person is modified as follows:

$$
\beta\left\{1-q^{S}(t)\right\} \theta_{t}^{S}\left\{1-q_{t}^{I}\right\} \theta_{t}^{I}=\beta \pi(t) \theta_{t}^{S} \theta_{t}^{I}
$$

with $\pi(t)=\left\{1-q^{S}(t)\right\}\left\{1-q_{t}^{I}\right\} \in[0 ; 1] . \pi(t)$ changes the chances that a susceptible person will encounter an infected person or vice versa, which is called a transmission modifier. The result is a new SIR model with a time-varying transmission rate modifier

$$
\left\{\frac{d \theta_{t}^{S}}{d t}=-\beta \pi(t) \theta_{t}^{S} \theta_{t}^{I} \frac{d \theta_{t}^{I}}{d t}=\beta \pi(t) \theta_{t}^{S} \theta_{t}^{I} \quad \frac{d \theta_{t}^{R}}{d t}=\quad \gamma \pi(t) \theta_{t}^{I}-\gamma \theta_{t}^{I}\right.
$$

where $\beta \pi(t)$ defines a realistic transmission rate reflecting the measures currently applied at all levels. We assume that the chances of being susceptible at the population level and the chances of being infected at the population level remain the same, but the chances of a susceptible person meeting an infected person are reduced by $\pi(t)$. A possible choice of $\pi(t)$ may be a staggered function that reflects the public health measures.

Since the dates of government policies are close to each other, it would be difficult to measure the individual effect of these on the spread of the virus. Thus, we define $\pi(t)$ as an exponential function 


$$
\pi(t)=\{\exp \exp (-\alpha t) \text { if } t \in T 1 \text { if } t \notin T
$$

where $T$ is the period during which policies were adopted.

L. Wang et al. (2020) proposed the resolution of the model through a fourth-order Runge-Kutta approximation (RK4). In our approach, we propose a preliminary estimate of the parameters $\beta>0$ and $\gamma>0$ representing respectively the rate of spread of the disease and the rate of remission by ordinary differential equations (ODE). They are determined by solving the equation below:

$$
\left\{\frac{d S(t)}{d t}=-\beta \times \frac{S(t) I(t)}{N} \frac{d I(t)}{d t}=\beta \times \frac{I(t) S(t)}{d t} \frac{d R(t)}{d t}=\quad \gamma I(t)-\gamma I(t)\right.
$$

where $S(t), I(t)$ and $R(t)$ represents respectively the populations of susceptible, infected and cured at time $t . \frac{S(t) I(t)}{N}$ is the probability of an encounter between a healthy person and an infected person. Once determined, these parameters are then introduced into the system.

\section{METHODOLOGY}

\section{Data collection}

The database was created from the publications of the reports on the epidemiological situation (SitRep) of COVID-19 issued by the Ministry of Health of Burkina Faso. The reports were prepared and published on a daily basis. These present daily cumulative data on infections, recoveries and deaths. These data can be obtained on the dashboard of the National Observatory of Health and Population (ONSP) of Burkina Faso (http://onsp.ms.sante.gov.bf/donnees/covid19). The data of the analysis cover the first days of the epidemic in Burkina Faso, the period from March 9 to April 30, 2020. The cumulative number of infectious and removed are presented in Table 1.

\section{Modeling}

We simulated our data by using a priori distributions parameters of the model:

$\theta_{0}^{I} \sim \operatorname{Beta}\left(1,\left(Y_{1}^{I}\right)^{-1}\right) ; \theta_{0}^{S} \sim \operatorname{Beta}\left(1,\left(Y_{1}^{S}\right)^{-1}\right) ; \theta_{0}^{R}=1-\theta_{0}^{S}-\theta_{0}^{I}$; R0 = 3.61 (Y. Liu et al., 2020); $\quad k \sim \operatorname{Gamma}(2,0.0001) ; \quad \lambda^{I} \sim \operatorname{Gamma}(2,0.0001) ; \quad \lambda^{R} \sim \operatorname{Gamma}(2,0.0001)$ (Mkhatshwa \& Mummert, 2011) with $s d(\beta)=s d(\gamma)=0.1$.

The data were analyzed using R software (version 3.6.0). We used the optim() function from the package deSolve (Soetaert et al., 2010) to solve ordinary differential equations. The package eSIR was used to run the MCMC simulation. This package was developed by Lilli Wang and her team (L. Wang et al., 2020) and is freely downloadable on GitHub.com/ lilywang1988/eSIR.

Our models were run on 500,000 replications including 200,000 for burn in. 


\section{RESULTS/FINDINGS}

On April 30, 2020, 649 persons were affected by the disease. Among them, 44 were deceased and 517 cured. The evolution of cases, cured and deaths is presented in Figure 1.

The following figures represent the estimation of the proportion of infected people. The black dots represent the actual values used for estimation and the blue vertical line represents the time where observed data were no longer feeding the model. The shaded colors represent the 95\% credible interval of the estimations $\theta_{t}^{I}$ and $\theta_{t}^{R}$ when data were available or the predicted proportions $Y_{t}^{I}$ and $Y_{t}^{R}$ when data were not available. The solid lines represent the point estimation (posterior mean and median).

Figure 2 presents the results of the estimation of COVID-19 infection rates from May 2020 to September 2020. The model estimates very low values-less than $0.01 \%$ of the population. However, when it comes to prediction, the estimations are characterized by high variability. Then, the credibility interval stands within zero and $20 \%$ of the population. Therefore, the model is showing a regular pattern where the situation will keep growing until a peak in July 2020. The number of daily cases will then be decreasing. While the median posterior estimation is still very low and unobservable in the graph, we observe that the exceptionally high values raise the mean posterior which can reach a maximum of $4 \%$ of the population. The key turning points are represented by the vertical lines.

The proportion of deaths due to COVID-19 is then presented in Figure 3. Once again, the estimations fit very well with the data. However, the predictions lead to very large credible intervals which will not help regarding interpretation.

When considering the public health measures to limit the spread of the disease, the model shows a different pattern. Figure 4 describes the evolution of the estimated values as well as the forecasts. In this case, the changing points, represented by the vertical lines, happen during the estimation period. This shows that the measures permit to contain the epidemic and to lead to its end. The infection rates remain very low $\left(1 \cdot 10^{-5} \approx 20\right.$ cases $)$. Then, the epidemic is predicted to end in early August 2020.

The observed situation is confirmed by the graph of the removed compartment (Figure 5).

\section{DISCUSSION}

In this paper, we adapted an original approach for infectious disease modelling to Burkina Faso data. Additionally, we proposed a more objective approach to determine the model parameters as the parameters found in the literature, measured in other contexts, are not always reliable. Our approach involves fitting a simple ordinary differential equation (ODE) susceptibleinfectious-removed (SIR) model with parameters we believe in and where the remaining values will be fitted using available data. The obtained new parameters are then used as prior values in the statistical model.

We proposed two models in this paper. The first one just relies on the published data without considering any external information. This approach leads to very imprecise predictions where decision making will be very hard. The second model considers the key public health measures 
which were undertaken in Burkina Faso at the beginning of the pandemic i.e., we considered the school closure on March 16, the curfew and the border closure on March 21. This new approach presents more accurate results. The one-year forecast is usable for decision making.

The approach relies on strong mathematical modelling theory and incorporates high flexibility. The strong mathematical theory is first related to the SIR dynamic modelling which is still considered as one of the more reliable approaches to model and forecast infectious diseases (Wearing et al., 2005). However, the ODE based SIR models rely on very strong assumptions related to the model parameters. Dealing with assumptions in a modelling and simulation exercise is key (Eriksson et al., 2009). The statistical approach uses the observed data to estimate the parameters and to make predictions. This reduces the number and the importance of the assumptions which are made at the beginning. Additionally, the Bayesian method considers the model parameters, not as fixed values but as randomly distributed, according to defined probability distributions. This much more reduces the importance of assumptions which can be made.

The third strength of such a modelling approach is the flexibility. In fact, the model incorporates several phases of the epidemic presented as public health measures which play as game changers in the course of the epidemic. Such flexibility is recommended for early-stage estimations where the information is scarce and where every data needs to be accounted for. Finally, the approach permits early estimation of the epidemic in Burkina Faso.

Just after the first cases in the country, an expert committee was set up to propose predictions and forecasts, and to give support for decision making. Only ODE type models could be proposed with very strong assumptions. The parameter values such as the disease infectiousness were adjusted on a daily basis according to the new information collected. We could propose statistical approaches only two to three months after the first case. This approach would have been very helpful in this process.

One could consider that regarding the current developments of the COVID-19 epidemics in the world, such a paper is useless. However, it aims to increase the knowledge and the practices in African countries. In fact, we are aiming at improving our readiness for major health emergencies. This readiness includes the ability to generate biomathematical and biostatistical evidence. Mohamadou et al. (2020) proposed a comprehensive review of mathematical models, artificial intelligence solutions as well as datasets used to study, predict or manage the COVID19 pandemic. In their vast review, we could not find any sub-Saharan African contribution. We are seeing this current paper as a pedagogical tool for young African researchers.

The current challenge in COVID-19 modelling concerns the different epidemic waves (Nesteruk, 2020). Several situations are influencing the epidemic curve. The experience has shown that the strong assumption of acquired immunity after cure is not valuable. Additionally, we can list the public health measures, the virus variants of interest and the vaccination. Some also consider some external elements as climatic seasons, public events (electoral campaigns and riots) in African countries. We are now aiming at including all these aspects to explain the disease evolution and to propose forecasting with several scenarios. This Bayesian approach will be adapted purposely. 


\section{CONCLUSION}

We have proposed estimations of the early period of the COVID-19 pandemic in Burkina Faso. The models were based on the classical SIR approach with Bayesian estimation. We then run a prediction of the proportion of infected and the proportion of removed for the following 12 months. Two approaches were proposed: the first is just based on the epidemiologic data (number of cases and number of removed by day); the second model considers public health measures which were undertaken to reduce the epidemic.

The approach is very useful to quickly set the situation of an epidemic and make decisions. This will be adapted for the current case of COVID-19 epidemic, around two years after its apparition.

\section{REFERENCES}

Erik, Volz, Marc, Baguelin, Sangeeta, Bhatia, Adhiratha, Booyasiri, Anne, Cori, Zulma, Cucunuba, Gina, Cuomo-Dannenburg, Christl A., Donnelly, Alaria, Dorigatti, Rich, FitzJohn, Han, Fu, Katy, Gaythorpe, Azra, Ghani, Arran, Hamlet, Wes, Hinsley, Natsuko, Imai, Daniel, Laydon, Gemma, Nedjati-Gilani, Lucy, Okell, ... Neil M., Fergusson. (2020). Report 5: Phylogenetic analysis of SARS-CoV-2 (No. 5; Imperial College London COVID-19 Response Team). Imperial College. https://doi.org/10.25561/77169

Eriksson, H., Morin, M., Ekberg, J., Jenvald, J., \& Timpka, T. (2009). Assumptions management in simulation of infectious disease outbreaks. AMIA ... Annual Symposium Proceedings / AMIA Symposium. AMIA Symposium, 2009, 173-177.

Ferguson, N., Laydon, D., Nedjati Gilani, G., Imai, N., Ainslie, K., Baguelin, M., Bhatia, S., Boonyasiri, A., Cucunuba Perez, Z., Cuomo-Dannenburg, G., Dighe, A., Dorigatti, I., Fu, H., Gaythorpe, K., Green, W., Hamlet, A., Hinsley, W., Okell, L., Van Elsland, S., ... Ghani, A. (2020). Report 9: Impact of non-pharmaceutical interventions (NPIs) to reduce COVID19 mortality and healthcare demand [Report]. https://doi.org/10.25561/77482

GU, C., Jiang, W., Zhao, T., \& Zheng, B. (2020). Mathematical Recommendations to Fight Against COVID-19. SSRN Electronic Journal, 1-13. https://doi.org/10.2139/ssrn.3551006

Guiro, A., Koné, B., \& Ouaro, S. (2020). Mathematical Model of the Spread of the Coronavirus Disease 2019 (COVID-19) in Burkina Faso. Applied Mathematics, 11(11), 1204-1218. https://doi.org/10.4236/am.2020.1111082

Imai, N., Cori, A., Dorigatti, I., Baguelin, M., Donnelly, C. A., Riley, S., \& Fergusson, N. M. (2020). Report 3: Transmissibility of 2019-nCoV (No. 2; Imperial College London COVID-19 Response Team). Imperial College.

Imai, N., Dorigatti, I., Cori, A., Donnelly, C., Riley, S., \& Ferguson, N. M. (2020). Report 2: Estimating the potential total number of novel Coronavirus cases in Wuhan City, China. Annals of Biomedical Engineering, 48(January), 1-4.

Liu, Y., Gayle, A. A., Wilder-Smith, A., \& Rocklöv, J. (2020). The reproductive number of COVID-19 is higher compared to SARS coronavirus. Journal of Travel Medicine, 27(2), 1-4. https://doi.org/10.1093/jtm/taaa021

Liu, Z., Magal, P., Seydi, O., \& Webb, G. (2020). Understanding Unreported Cases in the COVID-19 Epidemic Outbreak in Wuhan, China, and the Importance of Major Public Health Interventions. Biology, 9(3), 50. https://doi.org/10.3390/biology9030050 
Mkhatshwa, T., \& Mummert, A. (2011). Modeling super-spreading events for infectious diseases: Case study SARS. IAENG International Journal of Applied Mathematics, 41(2), 82-88.

Mohamadou, Y., Halidou, A., \& Tiam, P. (2020). Mohamadou-2020-A review of mathematical modeli.pdf. 3913-3925.

Nesteruk, I. (2020). Waves of COVID-19 pandemic. Detection and SIR simulations (preprint). MedRxiv, March 2021, 2020.08.03.20167098.

Rabajante, J. F. (2020). Insights from early mathematical models of 2019-nCoV acute respiratory disease (COVID-19) dynamics. ArXiv:2002.05296 [q-Bio]. http://arxiv.org/abs/2002.05296

Soetaert, K., Petzoldt, T., \& Setzer, R. W. (2010). Solving Differential Equations in R: Package deSolve. Journal of Statistical Software, 33, 1-25. https://doi.org/10.18637/jss.v033.i09

Sohrabi, C., Alsafi, Z., O’Neill, N., Khan, M., Kerwan, A., Al-Jabir, A., Iosifidis, C., \& Agha, R. (2020). World Health Organization declares global emergency: A review of the 2019 novel coronavirus (COVID-19). International Journal of Surgery, 76(February), 71-76. https://doi.org/10.1016/j.ijsu.2020.02.034

UNDP. (2020). Human Development Reports. http://hdr.undp.org/en/countries/profiles/BFA\#

Wang, C., Horby, P. W., Hayden, F. G., \& Gao, G. F. (2020). A novel coronavirus outbreak of global health concern. The Lancet, 395(10223), 470-473. https://doi.org/10.1016/S0140-6736(20)30185-9

Wang, L., Zhou, Y., He, J., Zhu, B., Wang, F., Tang, L., Kleinsasser, M., Barker, D., Eisenberg, M. C., \& Song, P. X. (2020). An epidemiological forecast model and software assessing interventions on COVID-19 epidemic in China. Journal of Data Science, 18(3), 446-454.

Wearing, H. J., Rohani, P., \& Keeling, M. J. (2005). Appropriate models for the management of infectious diseases. PLoS Medicine, 2(7), 0621-0627. https://doi.org/10.1371/journal.pmed.0020174

Zio, S., Tougri, I., \& Lamien, B. (2020). Propagation du COVID-19 au Burkina Faso: Modélisation Bayésienne et Quantification des incertitudes: première approche. Ecole Polytechnique de Ouagadougou (EPO) Ouagadougou.

Thiombiano, A. S. (2020, March 9). Burkina: Un couple testé positif au coronavirus. L'Actualité du Burkina Faso 24h/24. https://www.burkina24.com/2020/03/09/burkinaun-couple-teste-positif-au-coronavirus/

B24, R. (2020, March 14). Coronavirus (COVID-19): 7 cas confirmés au Burkina. L'Actualité du Burkina Faso 24h/24. https://www.burkina24.com/2020/03/14/coronavirus-covid-19-7-cas-confirmes-auburkina/

lefaso.net. (2020, March 21). Coronavirus au Burkina: Un couvre-feu de 19h à $5 \mathrm{~h}$ du matin instauré pour compter du 21 mars 2020 à minuit - leFaso.net [Infos]. lefaso.net. https://lefaso.net/spip.php?article95627

Kindo, N. (2020, March 24). COVID-19 à Ouagadougou: Fermeture des marchés et yaars à compter du 26 mars 2020. L'Actualité du Burkina Faso 24h/24. https://www.burkina24.com/2020/03/24/covid-19-a-ouagadougou-fermeture-desmarches-et-yaars-a-compter-du-26-mars-2020/

AIB. (2020, March 27). Burkina: Huit villes touchées par Covid-19 mises en quarantaine [Infos]. Aouaga.Com. http://news.aouaga.com/h/128452.html 


\section{APPENDIX}

Evolution of cases, deaths and recovery during the early phase of COVID-19 outbreak in Burkina Faso

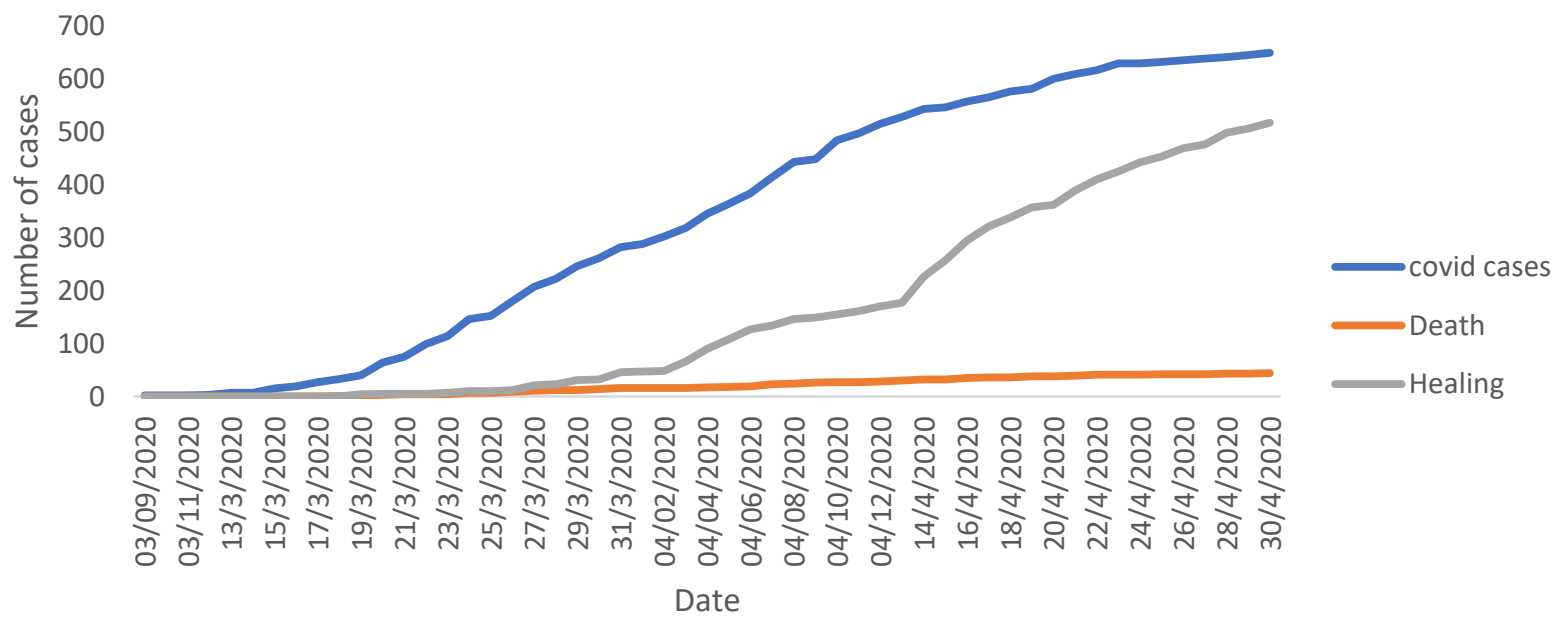

Figure 1: Evolution of cases, deaths and recovery during the early phase of COVID-19 outbreak in Burkina Faso

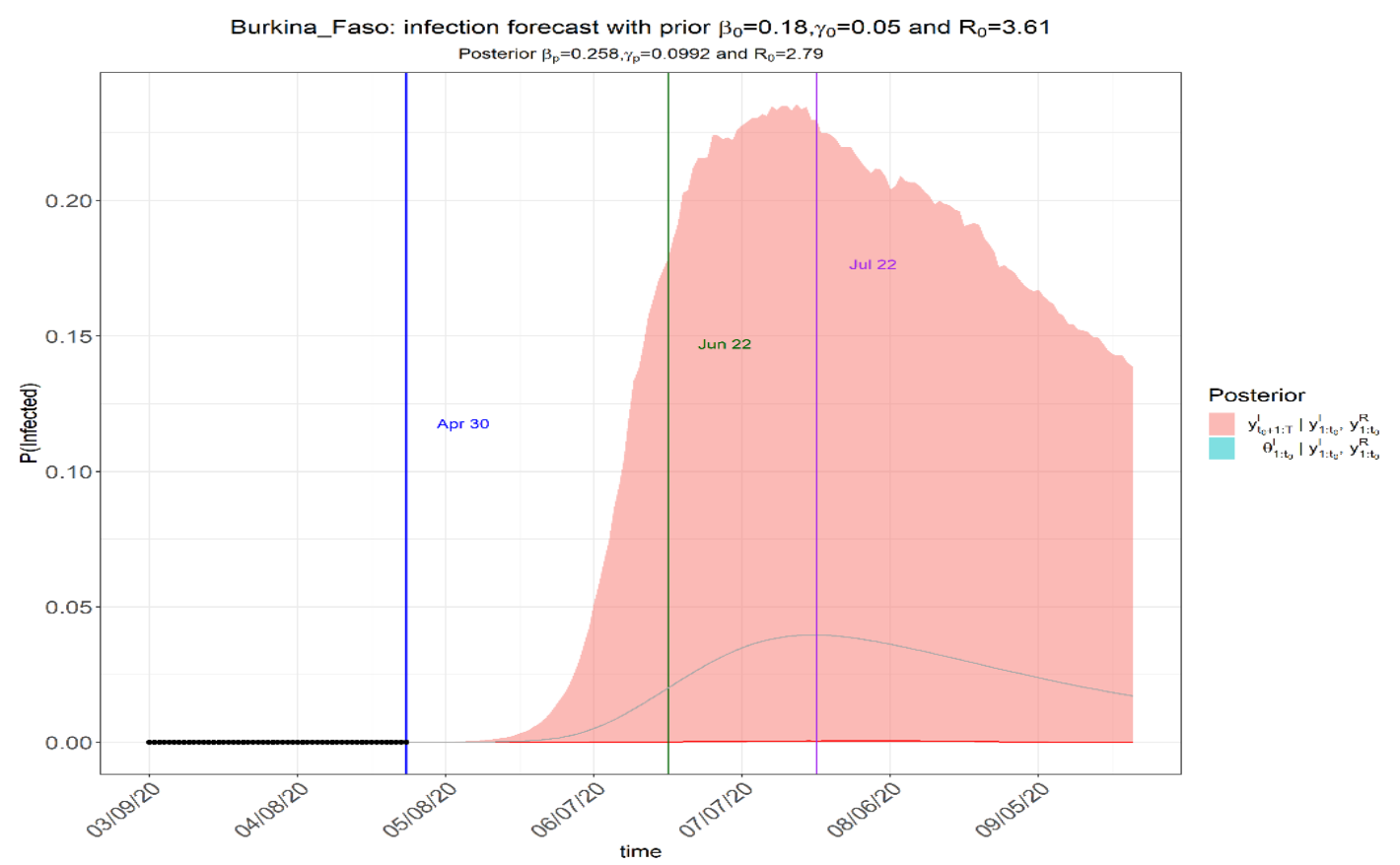

Figure 2: Estimation and forecasting of COVID-19 infection rate in Burkina Faso 


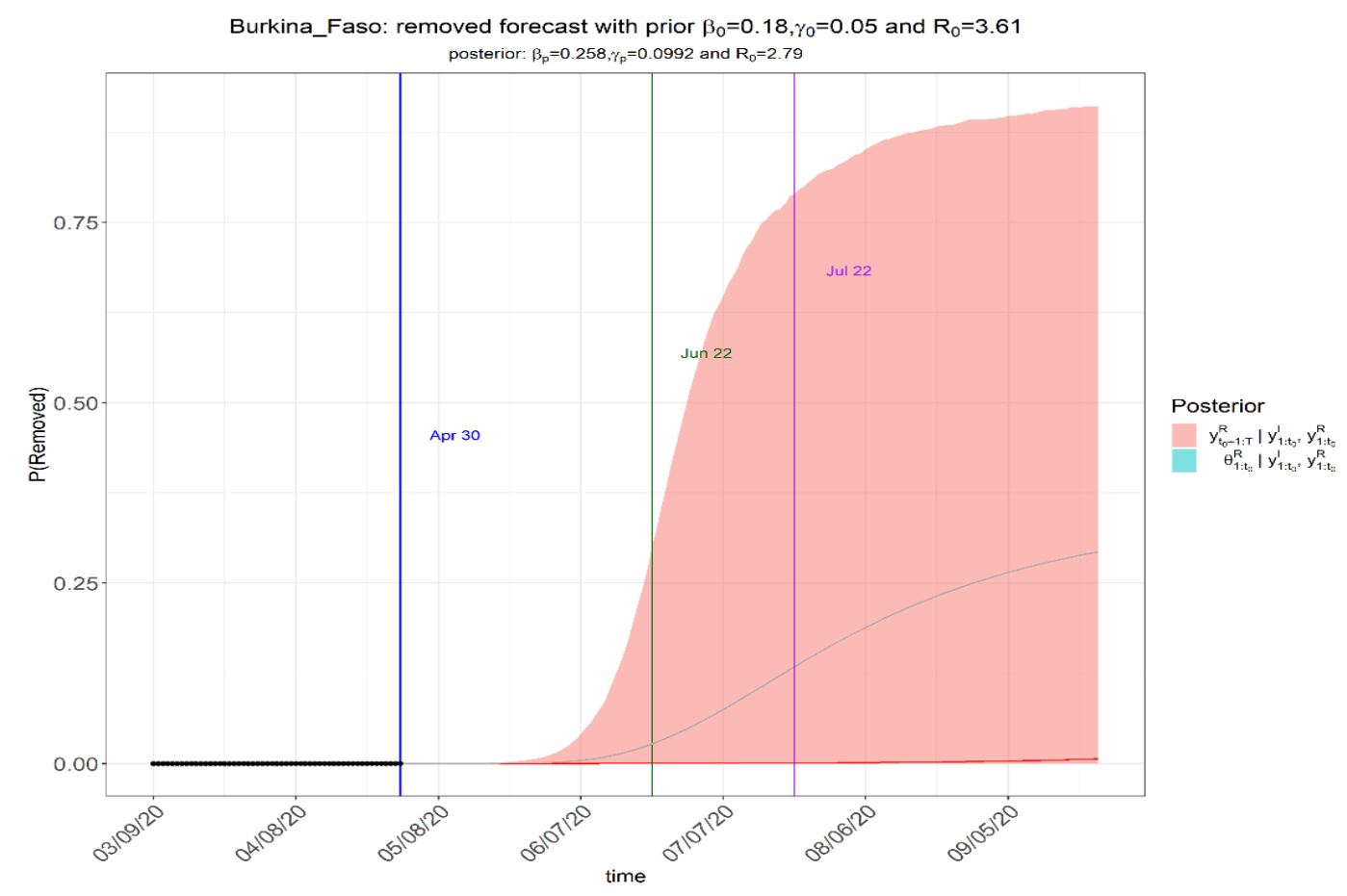

Figure 3: Estimation and forecasting of the proportion of the population removed after COVID-19 infection in Burkina Faso

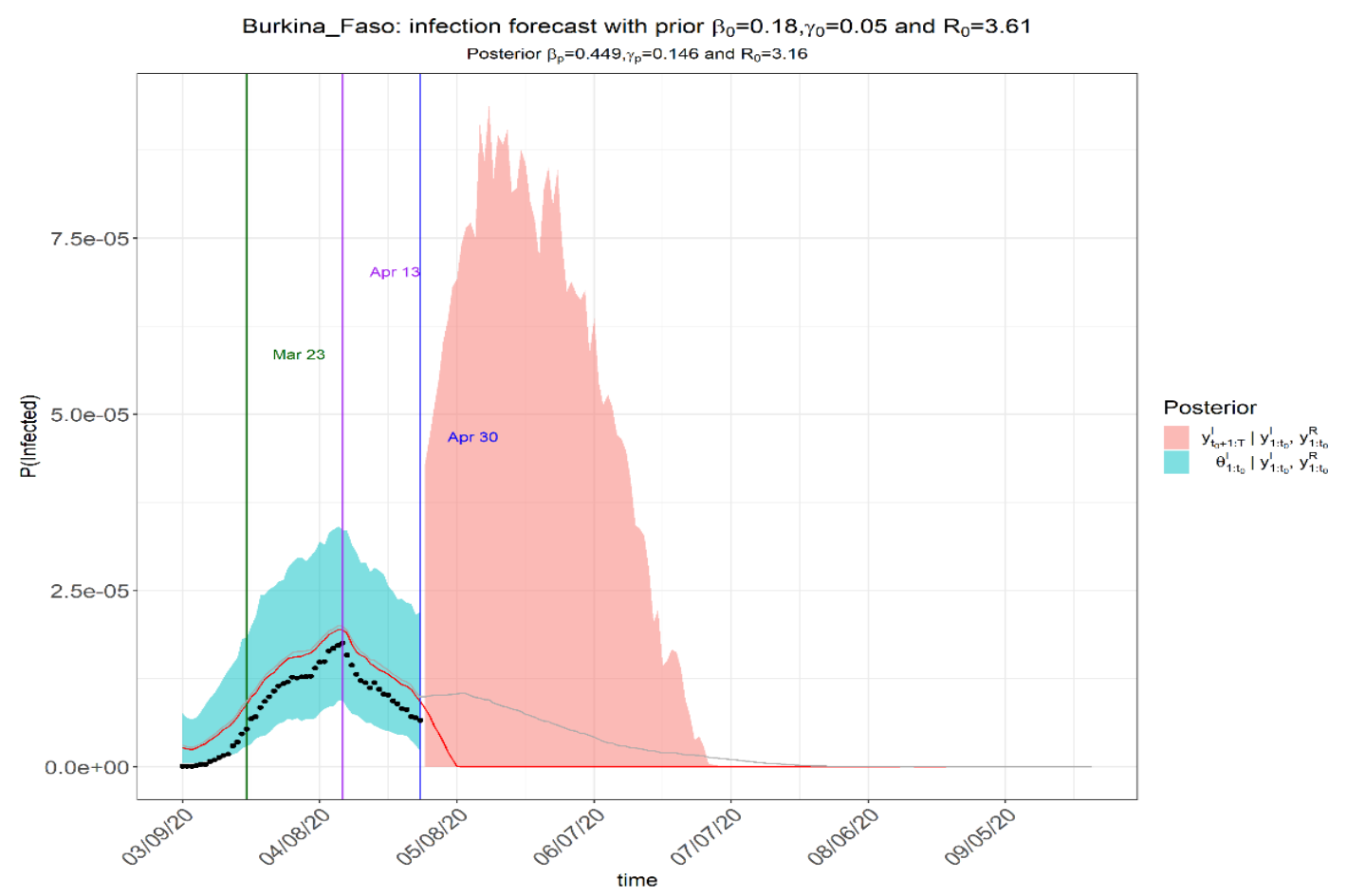

Figure 4: Estimation and forecasting of COVID-19 infection rate in Burkina Faso, considering public health measures 


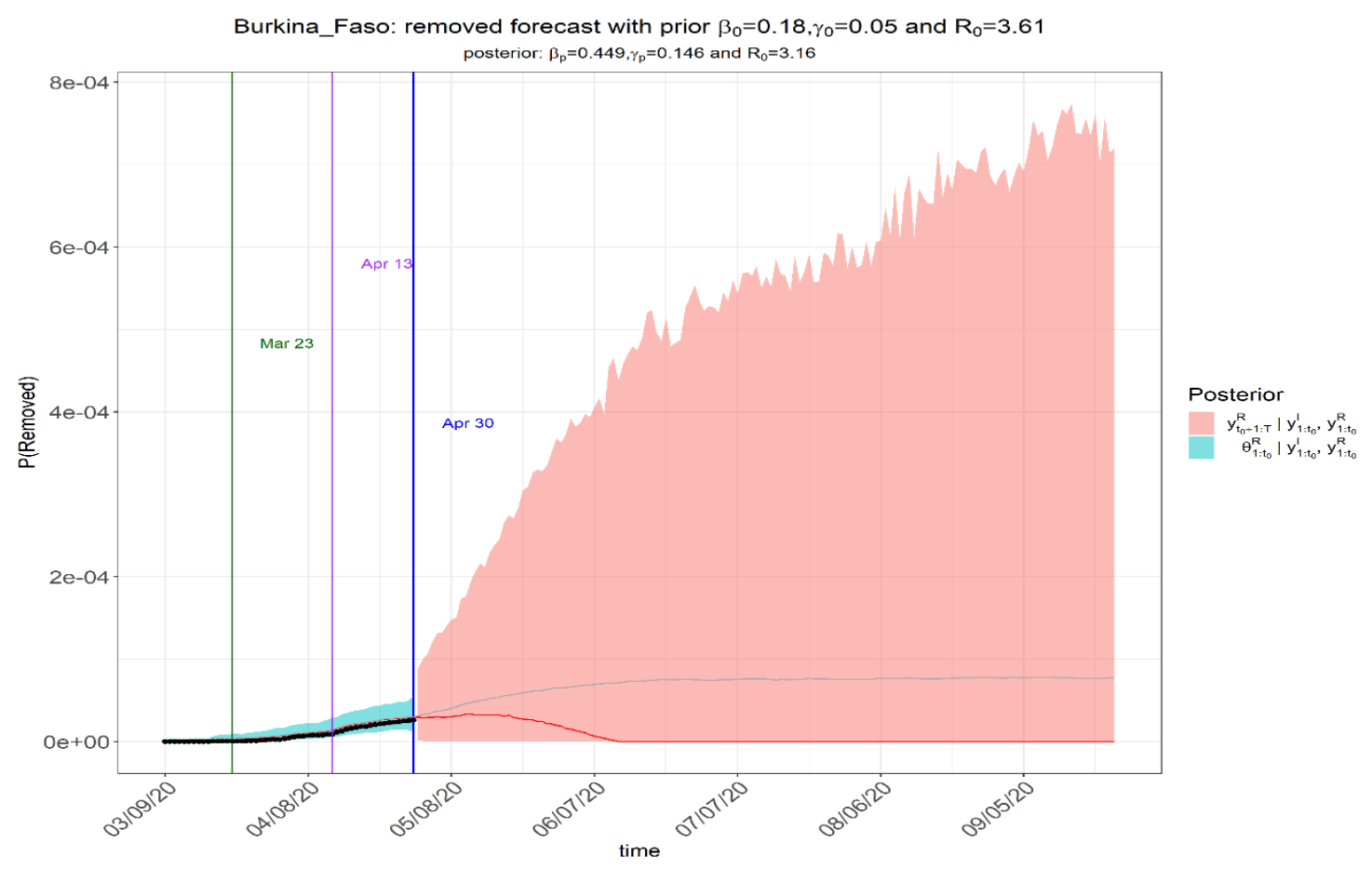

Figure 5: Estimation and forecasting of the proportion of the population removed after COVID-19 infection in Burkina Faso considering public health measures

Table 1: Cumulative number of infectious and removed used for the model

\begin{tabular}{|r|r|r|r|r|r|r|r|}
\hline \multicolumn{3}{|c|}{ INFECTIOUS } & \multicolumn{5}{|c|}{ REMOVED } \\
\hline 2 & 114 & 384 & 600 & 0 & 11 & 146 & 400 \\
\hline 2 & 146 & 414 & 609 & 0 & 17 & 157 & 428 \\
\hline 2 & 152 & 443 & 616 & 0 & 17 & 170 & 451 \\
\hline 3 & 180 & 448 & 629 & 0 & 21 & 175 & 466 \\
\hline 7 & 207 & 484 & 629 & 0 & 32 & 182 & 483 \\
\hline 7 & 222 & 497 & 632 & 0 & 35 & 188 & 495 \\
\hline 15 & 246 & 515 & 635 & 0 & 43 & 198 & 511 \\
\hline 19 & 261 & 528 & 638 & 0 & 46 & 207 & 518 \\
\hline 27 & 282 & 543 & 641 & 0 & 62 & 258 & 541 \\
\hline 33 & 288 & 546 & 645 & 1 & 63 & 289 & 549 \\
\hline 40 & 302 & 557 & 649 & 5 & 64 & 329 & 561 \\
\hline 64 & 318 & 565 & & 8 & 82 & 357 & \\
\hline 75 & 345 & 576 & & 9 & 107 & 374 & \\
\hline 99 & 364 & 581 & & 9 & 126 & 395 & \\
\hline
\end{tabular}

